EPJ Web of Conferences 36, 00018 (2012)

DOI: $10.1051 /$ epjconf/20123600018

(C) Owned by the authors, published by EDP Sciences, 2012

\title{
Formation time of hadronic resonances
}

\author{
Ivan Vitev ${ }^{1, a}$ \\ ${ }^{1}$ Los Alamos National Laboratory, Theoretical Division, Mail Stop B283 \\ Los Alamos, NM 87545, U.S.A.
}

\begin{abstract}
In heavy-ion collisions, formation time of hadrons of high transverse momentum can play a pivotal role in determining the perturbative dynamics of the final-state parton and particle system. We present methods to evaluate the formation times of light hadrons, hadronic resonances, open heavy flavor and quarkonia. Experimental implications of the short formation times of heavy particles are discussed in light of recent RHIC and LHC data.
\end{abstract}

\section{Introduction}

The discussion of particle formation and final-state dynamics in heavy-ion collisions, presented in this paper, follows ideas outlined in Refs. [1-4]. In contrast to proton-proton $(p+p)$ and proton-nucleus $(\mathrm{p}+\mathrm{A})$ reactions, in collisions of heavy nuclei $(\mathrm{A}+\mathrm{A})$ a strongly-interacting partonic medium of transverse size $\sim 2 R_{A}$, where $R_{A}$ is the nuclear radius, is created. The formation time of high- $p_{T}$ particles, which is determined by the particle production mechanism, must then be taken into account in simulations of in-medium interactions that modify the differential hard production cross sections and related hadron characteristics. Such effects are reflected, for example, in the nuclear modification ratio defined as follows:

$$
R_{A B}(\mathbf{p})=\frac{d \sigma_{A B}}{d y d^{2} \mathbf{p}} /\left(N_{\text {bin. }}^{A B}\right) \frac{d \sigma_{p p}}{d y d^{2} \mathbf{p}}
$$

In Eq. (1), $\sigma_{A B}$ refers to the production cross-section in collisions between two heavy ions $\mathrm{A}$ and $\mathrm{B}$, $\sigma_{p p}$ refers to the cross-section in collisions between two protons and $N_{\text {bin. }}^{A B}$ refers to the number of binary nucleon-nucleon collisions in an $\mathrm{A}+\mathrm{B}$ reaction.

Let us start our discussion on the example of light hadrons or open heavy flavor, produced via parton fragmentation. If a parton of non-zero mass $m_{Q}$ fragments into a massive hadron $m_{h}$ and a secondary light parton,

$$
\left[p^{+}, \frac{m_{Q}^{2}}{2 p^{+}}, \mathbf{0}\right] \rightarrow\left[z p^{+}, \frac{\mathbf{k}^{2}+m_{h}^{2}}{2 z p^{+}}, \mathbf{k}\right]+\left[(1-z) p^{+}, \frac{\mathbf{k}^{2}}{2(1-z) p^{+}},-\mathbf{k}\right] .
$$

In Eq. (2), $p^{+}$is the large light cone momentum of the parton, $z$ is the light cone momentum fraction taken by the hadron and $|\mathbf{k}| \sim \Lambda_{Q C D} \sim 200 \mathrm{MeV}$ is the deviation from collinearity. We can estimate from the uncertainty principle for the variable conjugate to the non-conserved light cone momentum component $\Delta p^{-}=\left(p^{-}\right)_{f}-\left(p^{-}\right)_{i}$ :

$$
\Delta y^{+} \simeq \frac{1}{\Delta p^{-}}=\frac{2 z(1-z) p^{+}}{\mathbf{k}^{2}+(1-z) m_{h}^{2}-z(1-z) m_{Q}^{2}} .
$$

\footnotetext{
a e-mail: ivitev@lanl.gov
}

This is an Open Access article distributed under the terms of the Creative Commons Attribution License 2.0, which permits unrestricted use, distribution, and reproduction in any medium, provided the original work is properly cited. 

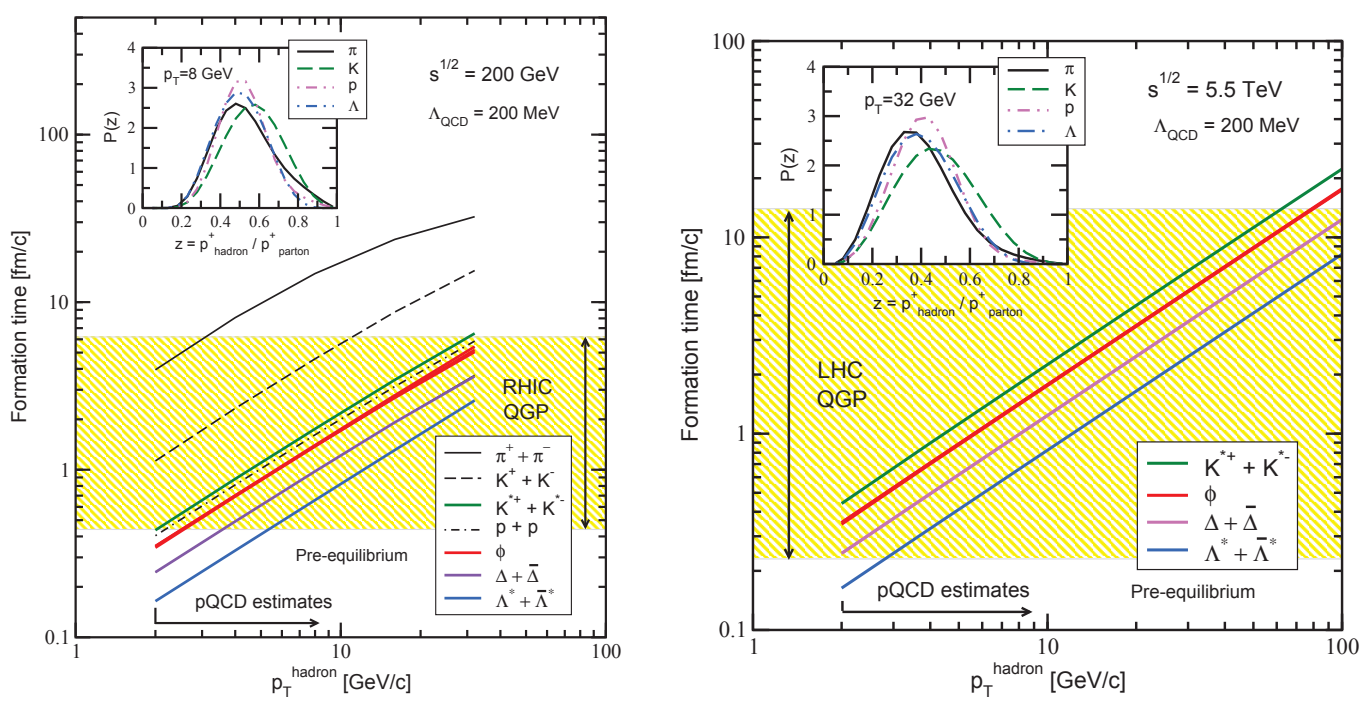

Fig. 1. Left panel: Transverse momentum dependence of leading hadron formation times at RHIC energies, $\sqrt{s}=$ $200 \mathrm{GeV}$. Results for both 'stable' particles and resonances are presented. Insert shows the meson and baryon light cone momentum fraction distributions $P(z)$ at a fixed momentum $p_{T}=8 \mathrm{GeV}$. Right panel: Transverse momentum dependence of heavy resonance formation times at LHC energies, $\sqrt{s}=5.5 \mathrm{TeV}$. Insert shows the meson and baryon distributions $P(z)$ at a fixed momentum $p_{T}=32 \mathrm{GeV}$.

With $\Delta y^{+}=\tau_{\text {form }}+\Delta z$ the formation time reads:

$$
\tau_{\text {form }}=\frac{\Delta y^{+}}{1+\beta_{Q}}, \quad \beta_{Q}=\frac{p_{Q}}{E_{Q}} .
$$

Clearly, the higher the energy, or $p_{T}$, and the lighter the hadron - the better the perturbative treatment of partonic energy loss in the quark-gluon plasma (QGP) prior to hadronization will be. For a $p_{T}=10 \mathrm{GeV}$ pion at mid-rapidity $\tau_{\text {form }} \approx 20 \mathrm{fm} \gg L_{T}^{\mathrm{QGP}} \simeq 2 R_{A}$, consistent with the jet quenching assumptions and the robust description of the nuclear modification factor $R_{A A}\left(p_{T}\right)$ versus $\sqrt{s_{N N}}, A$ and centrality $[5,6]$. A more detailed $p_{T}$ dependence of $\tau_{\text {form }}$, averaged over the momentum fraction $z$ distribution $P(z)$, for pions and kaons is shown in the left panel of Fig. 1.

In the following sections we will discuss examples when $\tau_{\text {form }} \ll L_{T}^{\mathrm{QGP}} \simeq 2 R_{A}$.

\section{Hadronic resonances}

Hadronic resonances are argued to play a central role in providing experimental evidence for partial chiral symmetry restoration in the deconfined quark-gluon phase produced in heavy ion collisions. Their lifetimes, which are comparable to the lifetime of the partonic plasma phase, make them a valuable tool to study medium modifications to the resonant state due to the chiral transition. The heavier, but still abundant, light and strange quark resonances $K^{*}, \phi, \Delta$ and $\Lambda^{*}$ have a large probability to be produced well within the plasma phase due to their short formation times [2].

Results for the mean formation time of hadronic resonances as a function of their $p_{T}$, compared to the estimated lifetime of the plasma at RHIC, are given in the left panel Figure 1. We note that at fixed transverse momentum $\left\langle\tau_{\text {form }}\right\rangle$ is controlled primarily by the particle mass. At higher $p_{T}$ there is a systematic bias toward larger mean values of $z$ but these only affect the formation time of the lightest particles, such as $\pi$ and $K$. We studied the validity of our assumption that we can get guidance for the formation times of resonant particles from the known $P(z)$ momentum fraction distributions for $\pi, K$, $p$, and $\Lambda$, shown in the insert of the figure for $p_{T}=8 \mathrm{GeV}$ at RHIC. $\tau_{\text {form }}$ for the $\phi$ meson was evaluated 


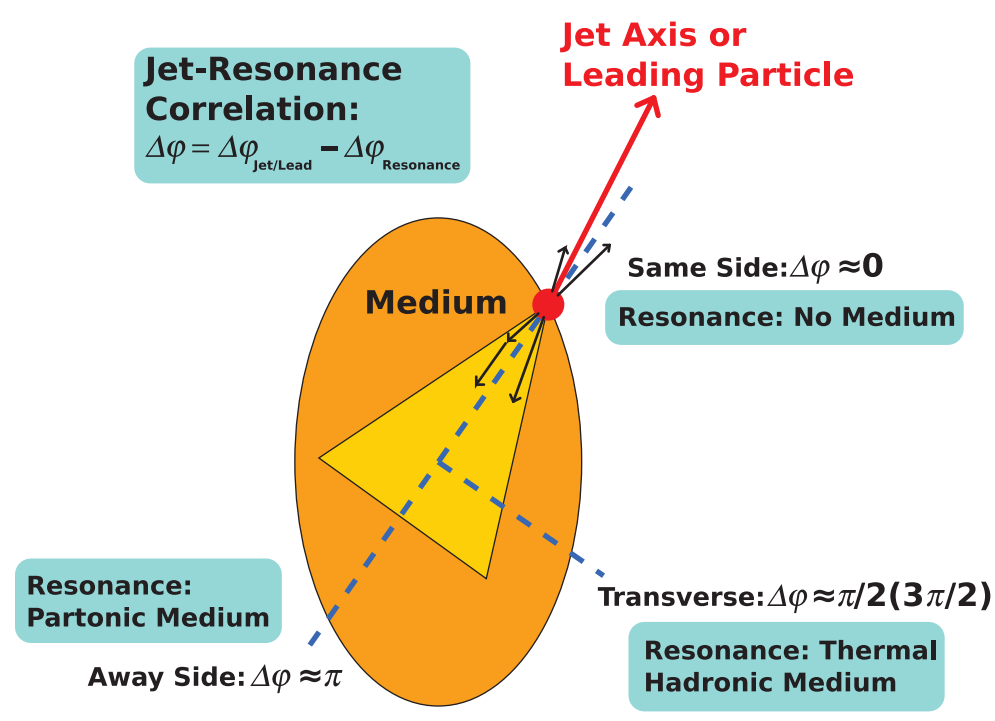

Fig. 2. Sketch of jet fragmentation into resonances $\left(\Lambda^{*}\right.$ is taken as an example for all hadronic resonances) in the medium created in a heavy-ion collision. Same-side correlations of resonances are not affected by the medium, whereas the away-side high $\mathrm{p}_{\mathrm{T}}$ resonance might be affected by the early (chiral restored) medium. Thermal resonances, which are affected by the late hadronic medium are at $\pi / 2$ with respect to the trigger particle.

with all available momentum fraction distributions and the uncertainty, presented in left panel of Fig 1 is not significant. In the perturbative regime, $p_{T}>$ few $\mathrm{GeV}$ light hadron production takes place largely at times that exceed the QGP lifetime and the observed nuclear modification is dominated by inelastic interactions of the parent parton [6]. Heavy particles, however, tend to be produced inside the plasma and would undergo broadening or dissociation. Depending on their $p_{T}$ range, $K^{*}, \phi, \Delta$ and $\Lambda$ are ideally suited to probe the early stages of the collision when the medium is hot and dense. Only high momentum resonances $\left(p_{T}>2 \mathrm{GeV} / \mathrm{c}\right)$ from the perturbative regime are considered, because low momentum bulk resonances from a thermal QGP phase will be formed later and are more likely, according to UrQMD simulation, to undergo large rescattering in the hadronic medium.

Triggering on a strongly interacting particle biases the partonic hard scattering toward the surface of the fireball and is an effective way of ensuring that the away-side resonance must traverse a medium of larger spatial extent, as shown in Fig. 2. The larger the trigger $p_{T}$, the more effective this technique is expected to be. The possibility of observing chiral symmetry restoration effects also depends on the resonance decay time of heavy resonances. Given their small vacuum decay widths, $\Gamma\left(K^{*}\right)=50.8 \mathrm{MeV}, \Gamma(\phi)=4.26 \mathrm{MeV}, \Gamma(\Delta) \approx 120 \mathrm{MeV}$ and $\Gamma\left(\Lambda^{*}\right)=15.6 \mathrm{MeV}$, for observation of any effect of partial chiral symmetry restoration a self-consistent reduction in their lifetime, related to the width broadening, is critical. Otherwise, even if the the partonic medium is radially co-moving with velocity as large as $v_{T} \simeq 0.7 c$, it is likely that high momentum resonances with moderate in-vacuum lifetime (e.g. $K^{*}, \Lambda^{*}$ ) will have their decay point boosted outside of the medium . This set the constraint that the medium-modified lifetime of the resonance can not exceed a few $\mathrm{fm} / \mathrm{c}$. Several model have been used [7] to calculate the broadening of the in-medium spectral functions and, although these calculations are based on interactions in a dense hadronic medium, parton-hadron duality can hopefully allow generalize the results to a partonic medium. It has been argued [7], on the example of the $\phi$ meson, that at $T=250 \mathrm{MeV}$ the shortening of the in-vacuum lifetime can be as large as a factor of ten.

The brief discussion of experimental results is limited to heavy ion collisions at RHIC and LHC. At present, experimental results suggest that there are no mass shifts or width broadening beyond 

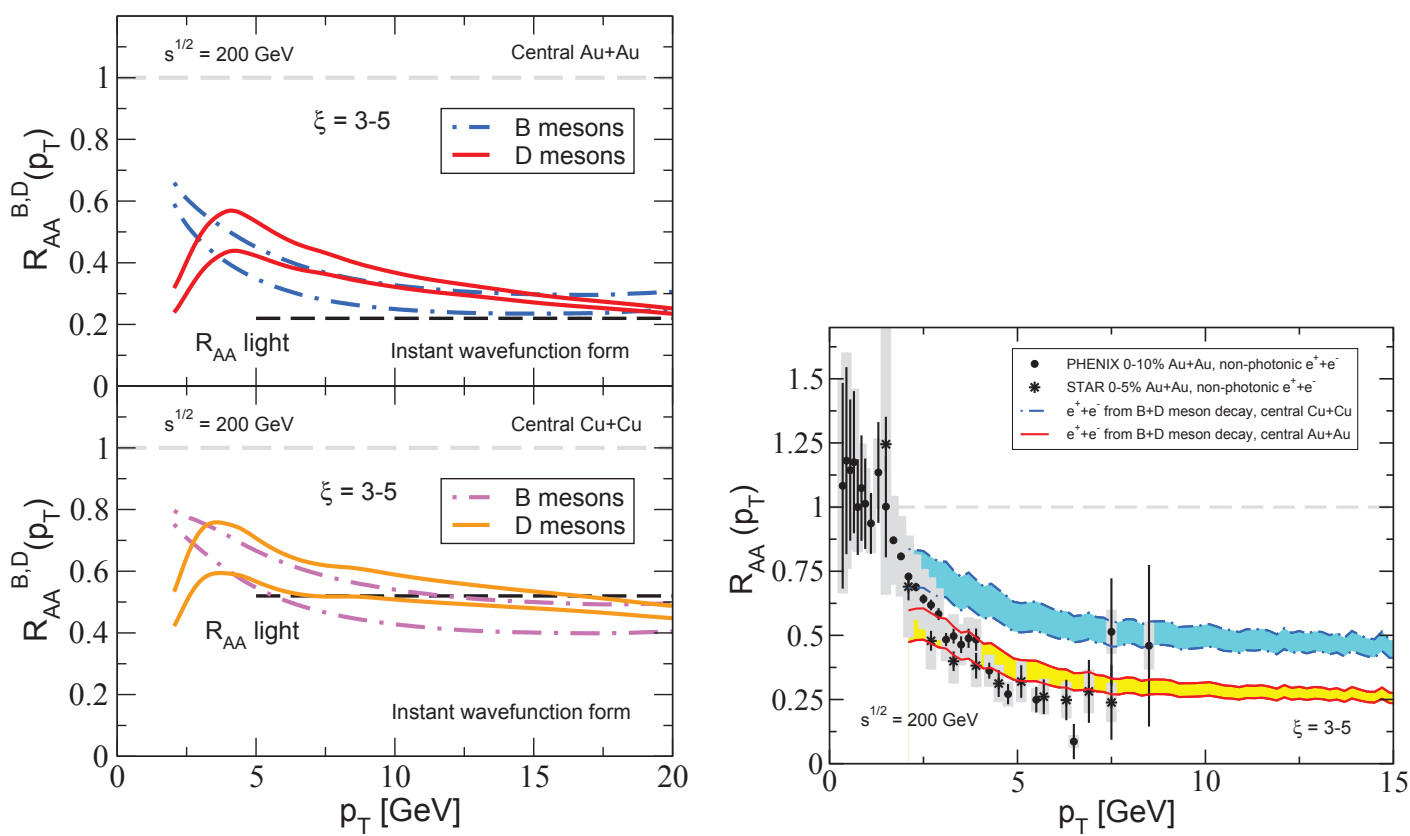

Fig. 3. Left panel: Suppression of $D$ and $B$ hadron production from meson dissociation and heavy quark quenching in central $\mathrm{Au}+\mathrm{Au}$ (top) and $\mathrm{Cu}+\mathrm{Cu}$ collisions (bottom) at $\sqrt{s_{N N}}=200 \mathrm{GeV}$ at RHIC. Right panel: Nuclear modification for the single non-photonic electrons in central $\mathrm{Au}+\mathrm{Au}$ and $\mathrm{Cu}+\mathrm{Cu}$ collisions at RHIC. Data is from PHENIX [12] and STAR [13] collaborations.

the detector resolution and systematics for both inclusive and hadron-tagged $\phi$ and $K^{0 *}$ in $\mathrm{Pb}+\mathrm{Pb}$ collisions in ALICE [8]. Similarly, the STAR collaboration at RHIC has studied resonance production in $\mathrm{Au}+\mathrm{Au}$ and $\mathrm{Cu}+\mathrm{Cu}$ production $[9,10] . \rho^{0}, \phi, \Sigma^{*}, \Lambda^{*}$. While a clear modification of the $\phi$ cross section is observed by the PHENIX collaboration, there is no modification of the mass and width of hadronic resonances.

\section{Open heavy flavor}

The formation time evaluation discussion, presented in Eq. (2) - Eq. (8) is also applicable to open heavy flavor production form the fragmentation of $c$ and $b$ quarks. In contrast to the light hadrons discussed in the Introduction, $B$ - and $D$-mesons of the same $p_{T}=10 \mathrm{GeV}$ have formation times $\tau_{\text {form }} \approx 1.5,0.4 \mathrm{fm}$, respectively, $\ll L_{T}^{\mathrm{QGP}}$. Therefore, at the finite $p_{T}$ range accessible at RHIC and LHC a conceptually different approach to the description of $D$ - and $B$-meson quenching in $\mathrm{A}+\mathrm{A}$ collisions is required, when compared to light hadrons.

By considering the propagation and interaction of a heavy-light quark-antiquark system in the QGP, we can evaluate the mean dissociation rate $\tau_{\text {diss }}\left(p_{T}, t\right)[1,3]$ of open heavy flavor mesons as a function of transverse momentum and time. Employing the calculated formation and dissociation rates, the concurrent processes of $c$ and $b$ quark fragmentation and $D$ and $B$ meson dissociation are described by the following set of rate equations:

$$
\begin{aligned}
& \partial_{t} f^{Q}\left(p_{T}, t\right)=-\frac{1}{\left\langle\tau_{\text {form }}\left(p_{T}, t\right)\right\rangle} f^{Q}\left(p_{T}, t\right)+\frac{1}{\left\langle\tau_{\text {diss }}\left(p_{T} / \bar{x}, t\right)\right\rangle} \int_{0}^{1} d x \frac{1}{x^{2}} \phi_{Q / H}(x) f^{H}\left(p_{T} / x, t\right), \\
& \partial_{t} f^{H}\left(p_{T}, t\right)=-\frac{1}{\left\langle\tau_{\text {diss }}\left(p_{T}, t\right)\right\rangle} f^{H}\left(p_{T}, t\right)+\frac{1}{\left\langle\tau_{\text {form }}\left(p_{T} / \bar{z}, t\right)\right\rangle} \int_{0}^{1} d z \frac{1}{z^{2}} D_{H / Q}(z) f^{Q}\left(p_{T} / z, t\right) .
\end{aligned}
$$


Here, $f^{Q}\left(p_{T}, t\right)\left(f^{H}\left(p_{T}, t\right)\right)$ is the differential cross section to find a quark $Q$ (hadron $H$ ) with a fixed rapidity $y$ and transverse momentum $p_{T}$, at time $t$. We have suppressed the rapidity dependence for clarity of notation. In Eqs. (5) and (6) $\phi_{Q / H}(x)$ is the distribution of the heavy quark Q inside the heavy meson $H$ and $D_{H / Q}(z)$ is the fragmentation function of the heavy quark Q into the heavy meson $H$. Note that the reason for which the asymptotic $t \rightarrow \infty$ solution will exhibit suppression of the cross sections is that both fragmentation and dissociation processes emulate energy loss by shifting the quarks/hadrons to lower momenta. Partonic energy loss can be incorporated in the form of quenched initial conditions $f^{Q}\left(p_{T}, t=0\right)=f_{\text {quench }}^{Q}\left(p_{T}\right), f^{H}\left(p_{T}, t=0\right)=0$.

We can integrate numerically the above set of coupled ordinary differential equations and use the same initial soft gluon rapidity density $d N^{g} / d y$ as in the simulations of light hadron quenching. The corresponding suppression of open heavy flavor in $\sqrt{s_{N N}}=200 \mathrm{GeV}$ central $\mathrm{Au}+\mathrm{Au}$ and $\mathrm{Cu}+\mathrm{Cu}$ collisions at RHIC is shown in the left panel of Fig. 3 For $D$ mesons the Cronin effect is clearly visible around $p_{T} \sim 4 \mathrm{GeV}$ and this is a notable difference from a previous study [1] where initial-state $k_{T}$ diffusion was not included. For $B$ mesons the change in heavy quark velocity $\beta_{Q}$, which controls the $Q \bar{q}(\bar{Q} q)$ broadening $\propto \beta_{Q}$ and dissociation, results in the characteristic rapid decrease in $R_{A A}^{B}\left(p_{T}\right)$ in this part of phase space. In both gold and copper reactions at RHIC the suppression $R_{A A}^{B} \approx R_{A A}^{D}$ for $p_{T}>4 \mathrm{GeV}$ and these approach the quenching of light hadrons for $p_{T}>10 \mathrm{GeV}$. It should be noted that while for the $D$ mesons we observe a transition from collisional dissociation to partonic energy loss in the studied kinematic domain, for B meson at RHIC the competing hadronic processes, Eqs. (5) and (6), still play the dominant role.

Our theoretical results, presented in the left panel of Fig. 3, are relevant to the future vertex detector upgrades at RHIC that will ensure direct and separate measurements of the $D$ and $B$ mesons. The STAR collaboration, however, has already made progress in identifying the contribution of $B$ mesons to the non-photonic electron spectrum [14]. Combined with the earlier $e^{+}+e^{-}$suppression measurements, this implies significant attenuation of $B$ meson production at transverse momenta as low as $5 \mathrm{GeV}$. This is one of the main predictions of the approach developed in $[1,3]$. The suppression of $D$ s in central $\mathrm{Pb}+\mathrm{Pb}$ collisions at the LHC [15] is also compatible with our theoretical predictions.

We use the PYTHIA event generator to simulate the full kinematics of the $D$ - and $B$-meson Dalitz decays in $\mathrm{p}+\mathrm{p}$ and $\mathrm{A}+\mathrm{A}$ reactions. The nuclear modification ratio $R_{A A}^{e}\left(p_{T}\right)$ of inclusive non-photonic electrons is presented in the right panel of Fig. 3 for $\sqrt{s_{N N}}=200 \mathrm{GeV}$ collisions at RHIC. Predictions for central $\mathrm{Cu}+\mathrm{Cu}$ collisions are also shown for comparison to upcoming STAR data. We remark that the Cronin effect included in our study changes the absolute scale of the differential heavy quark cross section at intermediate $p_{T}$. Consequently, to obtain a good description of the non-photonic $e^{+}+e^{-}$ quenching results from PHENIX [12] and STAR [13] we use $\xi=3-5$, approximately $50 \%$ larger than in our previous study [1] but still compatible with the enhancement of the parton broadening that comes from the power-law momentum transfer tails of the in-medium Moliere scattering. In $\mathrm{Cu}+\mathrm{Cu}$ reactions at RHIC the heavy meson dissociation mechanism provides a good description of the nonphotonic lepton suppression at mid [16] and forward rapidities [17].

\section{Quarkonia}

The study of hadron formation times has recently been extended to quarkonia [4]. To evaluate the baseline $J / \psi, \Upsilon, \cdots$ production cross section we used lowest order (LO) non-relativistic quantum chromodynamics (NRQCD). While more sophisticated approaches that incorporate higher order corrections and resummation of large logarithms exist, LO NRQCD gives an adequate description of the baseline quarkonium cross section to study nuclear effects.

The approach to estimating the formation time of quarkonium states differs considerably from the approach used for open heavy flavor [3] or light particles [2] that come from the fragmentation of a hard parton. In the latter case the formation time is inversely proportional to the virtuality of the parton decay and is governed by longitudinal dynamics. For quarkonia, the $Q \bar{Q}$ state is prepared instantly $\left(\sim 1 / \sqrt{p_{T}^{2}+M^{2}}\right)$ in the hard collision and subsequently expands to the spatial extent determined by the size of the asymptotic wavefunction. In this case all spatial directions are important. The velocity of 

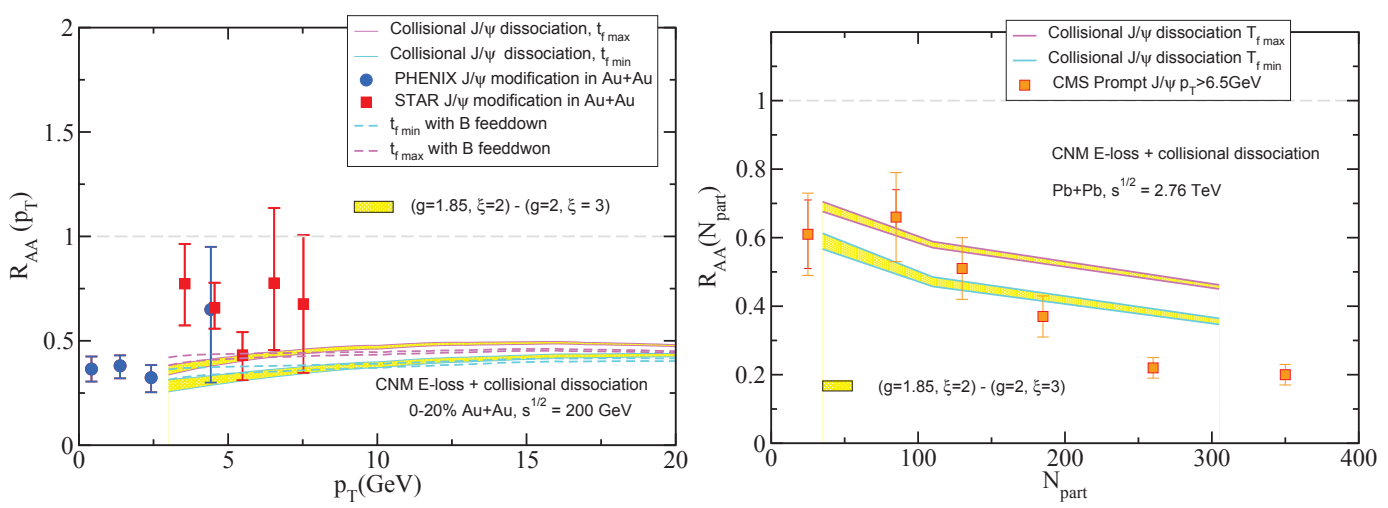

Fig. 4. Left panel: Theoretical model predictions for $J / \psi R_{A A}$ in $0-20 \%$ central RHIC Au+Au collisions at $\sqrt{S}=$ $0.2 \mathrm{TeV}$ [18]. Dashed curves include the $B \rightarrow J / \psi$ feed-down. Right panel: Expected $J / \psi$ suppression versus centrality $\left(N_{\text {part }}\right)$ at $\sqrt{S}=2.76 \mathrm{TeV}$. We compare the theoretical results to the CMS $R_{\mathrm{AA}}$ measurement [19].

the heavy quarks in the meson and a typical upper limit of the meson formation time can be evaluated as follows:

$$
\beta_{Q}=\sqrt{\frac{\kappa^{2}}{\kappa^{2}+m_{Q}^{2}}}, \quad t_{\text {rest frame }}^{\max }=\frac{a}{\beta_{Q}},
$$

where the typical momenta of heavy quarks inside the quarkonium states $\kappa$, the sizes of these stares $a$ can be obtained by solving the non-relativistic Schroedinger equation.

In this paper we are interested in high transverse momentum mesons, in which case there is a boost in the direction of propagation and, consequently, time dilation

$$
t_{\text {form }}^{\max }\left(p_{T}, \alpha\right)=\gamma t_{\text {rest frame }}^{\max }(\alpha)=\gamma \frac{a}{\beta_{Q}}, \quad \gamma=\frac{\sqrt{p_{T}^{2}+M^{2}}}{M} .
$$

For example, for the relevant formation time determined by the expansion of the $Q \bar{Q}$ state in a direction transverse to the direction of propagation the transverse size remains the same when boosted back to the laboratory frame but the velocity transforms by picking up a factor of $1 / \gamma$. Note that in Eq. (8) $|\mathbf{p}|=p_{T}$ since in this paper we work at mid-rapidity. The masses of the quarkonium states are denoted by $M$ and $\gamma$ is the meson boost factor. Since the formation process is non-perturbative and can not be modeled accurately, the values of $t_{\text {form }}$ obtained from Eq. 8 should be considered as an estimate of the upper bound. Therefore, in addition to calculating the final yields for $t_{\text {form }}=t_{\text {fmax }}=\frac{\gamma a}{\beta_{Q}}$, we also calculate the yields for $t_{\text {form }}=t_{\text {fmin }}=\frac{\gamma a}{2 \beta_{Q}}$ and the variation gives us an estimate of the uncertainty due to the uncertainty in the formation time. This does not change our results qualitatively.

Cold nuclear matter effects on high- $p_{T} J / \psi$ and $\Upsilon$ production have been discussed in [4]. Here, we are interested in $\mathrm{A}+\mathrm{A}$ reactions and neglect the Cronin effect but include initial-state cold nuclear matter energy loss and shadowing. Let us consider the nuclear modification factor for $J / \psi$ mesons. From the left panel of Fig. 4 we see that the measurement of $R_{A A}$ in RHIC Au+Au collisions [18] shows a suppression factor of about 0.6 at $p_{T} \sim 6 \mathrm{GeV}$. Even including the uncertainty in our model parameters, we obtain a somewhat higher suppression (for $p_{T} \sim 6 \mathrm{GeV}, R_{A A} \sim 0.35-0.45$ for $\mathrm{Au}+\mathrm{Au}$, than the one currently observed at RHIC. In Fig. 4, our results for the prompt yields of $J / \psi$ mesons are marked by upper and lower yellow bands corresponding to the upper $\left(t_{\text {form. }}^{\max }\right)$ and lower $\left(t_{\text {form. }}{ }^{\text {in }}=\right.$ $t_{\text {form. }}^{\max } / 2$ ) limits of our formation time estimate respectively. The bands themselves correspond to our estimate of the uncertainly in the sets of parameters that determine the coupling of the heavy quarks with the in-medium partons $[g=1.85, \xi=2$ (minimum considered coupling gives the upper limit of the yellow band) and $g=2, \xi=3$ (maximum considered coupling for the lower limit of the yellow band)]. The pronounced effect of the variation of the formation time can be intuitively seen as 
follows. The dissociation mechanism is operative only when the number of quarkonia is substantial, i.e. after $t_{\text {form. }}$. Since the upper limit for formation time of quarkonia can be on the order of several $\mathrm{fm} / \mathrm{c}$, the density of the medium at $t_{\text {form. }}^{\max }$ is reduced considerably due to Bjorken expansion, giving weaker dissociation and smaller suppression. This effect is more pronounced than the details of the coupling of heavy quarks to the in-medium partons. The RHIC experiments report suppression for the inclusive $J / \psi$ yield. For direct comparisons, we also show the $R_{A A}$ for the inclusive yields in the left panel of Fig. 4 with dashed lines. The $B$-meson yields for $\mathrm{p}+\mathrm{p}$ and $\mathrm{A}+\mathrm{A}$ collisions were taken from [3].

We now turn to $J / \psi$ production and modification at the LHC. In our formalism, we have approximated the quarkonium wavefunction by the vacuum wavefunction, which is valid if the thermal effects on the quarkonium wavefunctions are small. A higher suppression at LHC in comparison to the one predicted by our model could be the evidence for thermalization effects at the level of the quarkonium wavefunction. We leave a more detailed analysis of thermal effects on the wavefunctions for future work. Nevertheless, it is important to identify at what centrality the discrepancy between the present theoretical model predictions and the data may appear. In the right panel of Fig. 4 we show the $p_{T}$-averaged suppression,

$$
R_{A A}\left(N_{\text {part }}\right)=\frac{\int_{p_{\text {min. }}} d p_{T} R_{A A}\left(p_{T} ; N_{\text {part }}\right) \frac{d \sigma}{d y d p_{T}}}{\int_{p_{\text {min. }}} d p_{T} \frac{d \sigma}{d y d p_{T}}},
$$

of $J / \psi$ mesons versus centrality. A comparison to the CMS data [19] is shown in the right panel. The deviation between data and theory only appears for $N_{\text {part }}>200$.

\section{Conclusions}

In summary, we presented methods to evaluate the formation time of hadrons, hadronic resonances, open and closed heavy flavor at high transverse momentum. For heavy particles, $\tau_{\text {form }}$ can be considerably smaller than the size of the strongly-interacting plasma created in nucleus-nucleus reactions. This implies that final-state nuclear effects can be generated at the particle (as opposed to parton) level.

For the case of hadronic resonances, there is currently no evidence from experiments at RHIC and the LHC for broadening or mass shifts due to chiral symmetry restoration. This can also be a consequence of their small decay widths, which imply that the parent resonances will decay well outside of the medium, minimizing possible QGP and/or hadronic effects. For the case of open heavy flavor, collisional meson dissociation combined with parton energy loss has been successful in predicting the magnitude of the correlated $D$ - and $B$-meson suppression at RHIC and LHC energies and the quenching of the non-photonic electrons and muons coming form the semi-leptonic meson decay. For the case of quarkonia, the meson dissociation mechanism gives a good description of $J / \psi$ suppression at RHIC, implying a lack of thermal effects at the level of the quarkonium wavefunction. At the LHC, however, this mechanism underpredicts the magnitude of the suppression in the most central $\mathrm{Pb}+\mathrm{Pb}$ collisions. Advances in understanding the physics of jets in heavy ion collisions [20-22] can help extending the jet tagging techniques, currently applied to light hadrons and resonances to tagged heavy flavor to further elucidate particle production and propagation in strongly-interacting matter.

\section{References}

1. A. Adil and I. Vitev, Phys. Lett. B 649 (2007) 139 [hep-ph/0611109].

2. C. Markert, R. Bellwied and I. Vitev, Phys. Lett. B 669 (2008) 92 [arXiv:0807.1509 [nucl-th]].

3. R. Sharma, I. Vitev and B. -W. Zhang, Phys. Rev. C 80 (2009) 054902 [arXiv:0904.0032 [hep-ph]].

4. R. Sharma and I. Vitev, arXiv:1203.0329 [hep-ph].

5. M. Gyulassy, I. Vitev, X. -N. Wang and B. -W. Zhang, In *Hwa, R.C. (ed.) et al.: Quark gluon plasma* 123-191 [nucl-th/0302077]. 
6. I. Vitev, Phys. Lett. B 639 (2006) 38 [hep-ph/0603010].

7. L. Holt and K. Haglin, J. Phys. G 31, S245 (2005).

8. A. Knopspe [ALICE Collaboration], these proceedings.

9. M. Wada [STAR Collaboration], these proceedings.

10. M. M. Aggarwal et al. [STAR Collaboration], Phys. Rev. C 84 (2011) 034909 [arXiv:1006.1961 [nucl-ex]].

11. S. Campbell [PHENIX Collaboration], these proceedings.

12. A. Adare et al. [PHENIX Collaboration], Phys. Rev. Lett. 98, 172301 (2007).

13. B. I. Abelev et al. [STAR Collaboration], Phys. Rev. Lett. 98, 192301 (2007)

14. M. M. Aggarwal et al. [STAR Collaboration], Phys. Rev. Lett. 105 (2010) 202301 [arXiv:1007.1200 [nucl-ex]].

15. [ALICE Collaboration], arXiv:1203.2160 [nucl-ex].

16. A. Knospe, arXiv:1201.0242 [nucl-ex].

17. A. Adare, S. Afanasiev, C. Aidala, N. N. Ajitanand, Y. Akiba, H. Al-Bataineh, J. Alexander and K. Aoki et al., arXiv:1204.0754 [nucl-ex].

18. Z. Tang [STAR Collaboration], J. Phys. G 38, 124107 (2011) [arXiv:1107.0532 [hep-ex]].

19. S. Chatrchyan et al. [CMS Collaboration], [arXiv:1201.5069 [nucl-ex]].

20. R. B. Neufeld and I. Vitev, Phys. Rev. Lett. 108 (2012) 242001 arXiv:1202.5556 [hep-ph].

21. Y. He, I. Vitev and B. -W. Zhang, Phys. Lett. B 713 (2012) 224 [arXiv:1105.2566 [hep-ph]].

22. I. Vitev and B. -W. Zhang, Phys. Rev. Lett. 104 (2010) 132001 [arXiv:0910.1090 [hep-ph]]. 